\title{
miR-425 regulates lipophagy via SIRT1 to promote sorafenib resistance in liver cancer
}

\author{
GONGPING SUN, LIANG YANG, SHIBO WEI, HONGYUAN JIN, BOWEN LI and HANGYU LI \\ Department of General Surgery, The Fourth Affiliated Hospital, China Medical University, \\ Shenyang, Liaoning 110032, P.R. China
}

Received February 2, 2021; Accepted June 18, 2021

DOI: 10.3892/ol.2021.12956

\begin{abstract}
Liver cancer is one of the most malignant cancer, with poor outcomes and a high incidence rate, and current treatment approaches to prevent tumor progression and development remain unsatisfactory. Therefore, it is urgent to explore novel methods to inhibit tumor growth and metastasis. Autophagy is a highly conserved process associated with metastasis and drug resistance. Lipids are selectively recognized and degraded via autophagy; thus, autophagy is a crucial process to maintain tumor self-protection. MicroRNA (miR)-425 is a tumor-associated gene involved in liver cancer development that can induce cell proliferation and drug resistance. Using Cell Counting Kit- 8 assays, western blot analysis and immunofluorescence assays, the present study revealed that inhibition of miR-425 promoted lipophagy by mediating the autophagy process, which in turn helps to promote sorafenib resistance. Using a bioinformatics website, it was revealed that autophagy promoted lipophagy by targeting silent information regulator 2 homolog 1 (SIRT1). The results of luciferase reporter assays supported this finding, and rescue experiments provided additional evidence. Overall, the current results suggested that inhibition of miR-425 expression increased SIRT1 expression to promote lipophagy, leading to the inhibition of liver cancer cell proliferation.
\end{abstract}

\section{Introduction}

Liver cancer has the third highest rate of cancer-associated death worldwide (1). Hepatitis B virus infection is associated with most cases of liver cancer in China, accounting for

Correspondence to: Dr Hangyu Li, Department of General Surgery, The Fourth Affiliated Hospital, China Medical University, 4 Chongshandong Street, Shenyang, Liaoning 110032, P.R. China

E-mail: sj_li_hangyu@sina.com

Abbreviations: ncRNA, non-coding RNA; SIRT1, silent information regulator 2 homolog 1; NC, negative control; ATG, autophagy-related gene

Key words: autophagy, lipophagy, SIRT1, miR-425, liver cancer $\sim 85 \%$ of cases (2). Currently, the treatment of liver cancer mainly involves surgery, radiotherapy and chemotherapy. Although sorafenib has been established as an effective drug for advanced liver cancer, renal cell carcinoma and thyroid cancer, the number of patients with liver cancer exhibiting a complete response to sorafenib is small (3). In view of the emerging crisis of sorafenib resistance in hepatocellular carcinoma, further research on drug resistance is urgently required.

Autophagy is a cellular metabolic process in which cells degrade their own components through lysosomes or vacuoles to maintain normal physiological activities and homeostasis (4). In the process of tumor development, autophagy can allow the growth requirements of tumor cells to be met by degrading organelles and proteins (4). Altering the autophagy level in tumor cells is a new proposed approach for tumor therapy (5). Recently, a type of selective autophagy called lipophagy, through which lipids are selectively recognized and degraded, has been identified (6). Lipophagy serves an important role in regulating lipid metabolism and maintaining intracellular lipid homeostasis (6). Lipophagy is directly or indirectly regulated by genes, enzymes, transcription regulators and other factors $(7,8)$. Several studies support the idea that de novo lipogenesis in cancer cells is associated with chemoresistance at multiple levels $(6,7,9)$. The ability of lipophagy to respond to changes in nutrient supply allows the cell to alter lipid droplet (LD) metabolism to meet the cell's energy demands (10). Activation of lipophagy combined with standard chemotherapy can effectively decrease the LDs leading to improved chemotherapy efficiency (11). Consequently, targeting lipophagy to mediate LDs degradation may be a novel strategy to treat tumors and overcome drug resistance.

Non-coding RNAs (ncRNAs) have recently become recognized as important regulators in signaling pathways associated with drug resistance in liver cancer (12). Therefore, pharmacological targeting of these ncRNAs may constitute a novel strategy for reversing drug resistance (13). As an important protein involved in the nutrient-sensing pathway, along with the mammalian target of rapamycin protein and AMP-activated kinase in mammals, silent information regulator 2 homolog 1 (SIRT1) directly induces autophagy by deacetylating autophagy-related gene (ATG)5, ATG7 and LC3 (14). SIRT1 can also deacetylate FOXO to regulate the expression of autophagy regulatory molecules and modulate lipophagy $(15,16)$. However, the role of ncRNAs in regulating 
lipophagy in the context of drug resistance has not been reported. MicroRNA ( $\mathrm{miR} / \mathrm{miRNA}$ ) -425 is an oncogene that serves an important role in tumor progression and development (13); however, it has no clear mechanism in HCC drug resistance. The present study aimed to explore the mechanism by which miR-425 regulates lipophagy and the effect of miR-425 on sorafenib resistance.

\section{Materials and methods}

Chemicals and materials. miR-425 mimics and miR-425 inhibitor were purchased from Shanghai GenePharma Co. Ltd. Anti-LC3 (cat. no. 13394; Cell Signaling Technology, Inc.; $1: 1,000)$ and anti- $\beta$-actin antibodies were purchased from Abcam (cat. no. ab8226; 1;1,000). Bafilomycin A (BafA1; $25 \mathrm{nM}$; Beyotime Institute of Biotechnology) was used for treatment for $6 \mathrm{~h}$ at $37^{\circ} \mathrm{C}$.

Cell culture. HepG2 (CoBioer Biosciences Co., Ltd.; cat. no. CBP60199), Hep3B (CoBioer Biosciences Co., Ltd.; cat. no. CBP60197), PLC (CoBioer Biosciences Co., Ltd; cat. no. CBP60223), Huh7 (CoBioer Biosciences Co., Ltd.; cat. no. CBP60202) and MIHA (Hunan Fenghui Biotechnology Co., Ltd.; cat. no. CL0469) cells were cultured in DMEM (cat. no. 12430104; Invitrogen; Thermo Fisher Scientific, Inc.) supplemented with 10\% FBS (cat. no. 10091148; HyClone; Cytiva) and $1 \%$ penicillin-streptomycin solution (cat. no. 15140163; Gibco; Thermo Fisher Scientific, Inc.; ratio 9:1:1) at $37^{\circ} \mathrm{C}$ and $5 \% \mathrm{CO}_{2}$. Cross-contamination of the cell lines was excluded by short tandem repeat profiling $(17,18)$.

BODIPY staining. Cells were fixed with $4 \%$ paraformaldehyde for $10 \mathrm{~min}$ at room temperature and stained with $1 \mu \mathrm{g} / \mathrm{ml}$ BODIPY (cat. no. 493/503; Sigma-Aldrich; Merck KGaA) in PBS for $10 \mathrm{~min}$ at room temperature (11). After staining, coverslips were washed with PBS and mounted in a slide with Prolong Gold Antifade Reagent (Invitrogen; Thermo Fisher Scientific, Inc.). BODIPY stained cells were examined under an inverted confocal fluorescence microscope (Zeiss AG; magnification $\mathrm{x} 400)$.

Cell transfection. miR-425 mimics (5'-GUCAAAAAUGUC GAUAUGUCAUU-3'; $25 \mu \mathrm{M}$ ) and miR-425 inhibitor (5'-GGG GAGTTAGGATTAGGTC-3'; $50 \mu \mathrm{M}$ ) were purchased from Shanghai GenePharma Co., Ltd. Liver cancer cells were seeded in 6 -well plates at $5 \times 10^{5}$ cells/well. When the cells were $70 \%$ confluent, Lipofectamine ${ }^{\circledR} 3000$ (cat. no. L3000015; Invitrogen; Thermo Fisher Scientific, Inc.) was used for transfection with the miR-425 mimics, miR-425 inhibitor or the corresponding negative control (miR-NC 5'-CTCGCTTCG GCAGCACA-3' and si-NC: 5'-AACGCTTCACGAATTTGC GT- $\left.3^{\prime}\right)$ at $37^{\circ} \mathrm{C}$ and $5 \% \mathrm{CO}_{2}$. The culture medium was changed after $6 \mathrm{~h}$, and cells were harvested for PCR analysis after $48 \mathrm{~h}$, while western blotting was performed after $72 \mathrm{~h}$.

Immunofluorescence. Liver cancer cells were stained with anti-LC3 antibody (abcam; cat. no. ab225383) for $48 \mathrm{~h}$ and were then treated with miR-425 inhibitors and stained with $1 \mu \mathrm{g} / \mathrm{ml}$ BODIPY (cat. no. 493/503; Sigma-Aldrich; Merck $\mathrm{KGaA}$ ) in PBS for $10 \mathrm{~min}$ at room temperature. After induction of autophagy by miR-425 inhibitors, the number 61 of mCherry-positive cells was increased compared with that of untreated cells. Red fluorescent protein expression was quantitatively analyzed by ImageJ (v1.52) software. Cells were grown overnight on cover glasses, fixed with $4 \%$ paraformaldehyde $\left(37^{\circ} \mathrm{C}\right.$ and $\left.5 \% \mathrm{CO}_{2}\right)$ and sealed with $\mathrm{PBS}$ containing $1 \%$ bovine serum albumin (BSA; cat. no. ST023; Beyotime Institute of Biotechnology) for $1.5 \mathrm{~h}$ at room temperature. Stained samples were visualized using a Zeiss-LSM 510 fluorescence microscope (Zeiss AG; magnification, x400).

Reverse transcription-quantitative $(R T-q) P C R$. All detection of miRNA expression was performed by RT-qPCR. TRIzol ${ }^{\circledR}$ reagent (cat. no. 15596026; Invitrogen; Thermo Fisher Scientific, Inc.) was used to lyse cells for extraction of total RNA. Total RNA ( $2 \mu \mathrm{g})$ was reverse transcribed using a microRNA kit according to the manufacturer's protocol (cat. no. 4366596; Invitrogen; Thermo Fisher Scientific, Inc.). A $20-\mu 1$ final reaction mixture was used for qPCR amplification using SYBR Green PCR Master Mix (cat. no. 4309155; Thermo Fisher Scientific, Inc.). Pre-denaturation at $95^{\circ} \mathrm{C}$ for $3 \mathrm{~min}$, denaturation at $95^{\circ} \mathrm{C}$ for $30 \mathrm{sec}$, annealing at $55^{\circ} \mathrm{C}$ for $30 \mathrm{sec}$, extension at $72^{\circ} \mathrm{C}$ for $60 \mathrm{sec}$, for a total of 30 cycles, and after completion of the cycle, extension at $72^{\circ} \mathrm{C}$ for $5 \mathrm{~min}$. The target mRNA expression levels were normalized to the levels of $\beta$-actin, U6 was used as an reaction internal reference. All samples were repeated for 3 wells. RNA expression levels were calculated using the $2^{-\Delta \Delta \mathrm{Cq}}$ method (19). The primer sequences used were as follows: MiR-425 forward, 5'-TGCGCTCAG CAAACATTTATTG-3' and reverse, 5'-CCAGTGCAGGGT CCGAGGTATT-3'; U6 forward, 5'-CTCGCTTCGGCAGCA CA-3' and reverse, 5'-AACGCTTCACGAATTTGCGT-3'; SIRT1 forward, 5'-GTGCAGGTAGTTCCTCGGTG-3' and reverse, 5'-CACAACTCACAGCATGCACAA-3'; and $\beta$-actin forward, 5'-AGCGAGCATCCCCCAAAGTT-3' and reverse, 5'-GGGCACGAAGGCTCATCATT-3'; ATGL forward, 5'-GTGTCAGACGGCGAGAATG-3', reverse, 5'-TGGAGG GAGGGAGGGATG-3'

Western blot analysis. Cells were lysed in RIPA buffer (cat. no. P0013B; Beyotime Institute of Biotechnology) at $4^{\circ} \mathrm{C} 101$ for $2 \mathrm{~h}$. Supernatants were collected and the protein concentra- 102 tions were quantified by bicinchoninic acid (BCA) via using a 103 BCA protein assay kit (Beyotime Institute of Biotechnology). 104 Proteins (30 $\mu \mathrm{g} / \mathrm{lane})$ were separated via 10\% SDS-PAGE and 105 were then transferred onto PVDF membranes (cat. no. 88585; 106 Thermo Fisher Scientific, Inc.). Subsequently, membranes were 107 blocked in 5\% BSA for $2 \mathrm{~h}$ at $37^{\circ} \mathrm{C}$ and incubated with anti-LC3 108 (1:1,000), anti-ATGL (1:1,000) (Abcam; cat. no. ab207799) 109 or anti- $\beta$-actin $(1: 1,000$; cat. no. ab8226; Abcam) primary 110 antibodies overnight at $4^{\circ} \mathrm{C}$. After primary antibody incu- 111 bation, membranes were incubated with HRP-conjugated 112 secondary antibodies (cat. no. sc-2357; 1:5,000; Santa Cruz 113 Biotechnology, Inc). The proteins were visualized using an 114 enhanced chemiluminescence detection kit (Amersham; 115 Cytiva). The gray values of the protein bands were measured 116 using ImageJ (v1.52) software (National Institutes of Health). 117

Luciferase reporter assay. The SIRT1 3'UTR containing the 119 specific binding site for miR-425 was amplified by PCR. The 120 
3'UTR sequence containing a mutation in the specific binding site was also amplified, and then the sequences were inserted into the pre-miR-RB-REPORT vector and transfection into cells (Thermo Fisher Scientific, Inc.). Lipofectamine 3000 was used to transfection (Lipofectamine ${ }^{\circledR}$ 3000; cat. no. L3000015; Invitrogen; Thermo Fisher Scientific, Inc.). After $24 \mathrm{~h}$ of transfection with mimic-NC and miR-425 mimics, a luciferase assay system (cat. no. 16170; Thermo Fisher Scientific, Inc.) was used to perform the assay, and Renilla luciferase activity was used for normalization.

Cell Counting Kit-8 (CCK-8) assay. Cells (2,000 cells/well) were seeded in 96-well plates (Corning, Inc.) in DMEM with 10\% FBS (cat. no. 10091148; HyClone; Cytiva). Cells were then treated with sorafenib $\left(10 \mu \mathrm{M}, 37^{\circ} \mathrm{C}\right.$ and $\left.5 \% \mathrm{CO}_{2}\right)$. After culturing the cells for $48 \mathrm{~h}, \mathrm{CCK}-8$ solution (cat. no. C0037; Beyotime Institute of Biotechnology) was added to the cells for $30 \mathrm{~min}$ at room temperature. The absorbance was measured with an ELISA plate reader (Varioskan Flash; Thermo Fisher Scientific, Inc.) at $450 \mathrm{~nm}$.

Bioinformatic analysis. The Cancer Genome Atlas (TCGA) liver cancer datasets were obtained from https://portal.gdc.cancer. gov/. Gene Expression Omnibus (GEO) datasets (dataset nos. GSE93595 and GSE94550) $(20,21)$ were obtained from https:// www.ncbi.nlm.nih.gov/geo/. The gene expression matrix for the TCGA datasets was analyzed using R software v4.0.2 (limma package v3.44.3) $(22,23)$ using log fold-change $(F C)>0.6$ or $\log \mathrm{FC}<-0.6$ and $\mathrm{P}<0.05$ as the cut-off values, and the correlation between miR-425 and SIRT1 expression was evaluated using Pearson correlation analysis with SPSS (22.0; IBM, Corp.). GEO datasets were analyzed with GEO2R (v3.2.3) (Table SI) $(24,25)$, and Venn diagrams were generated with VennDiagram (v1.2.20) (24,25). The site for miR-425 binding with SIRT1 was predicted using StarBase 2.0 (http://starbase.sysu.edu.cn/).

Invasion and migration assays. Cells $\left(2 \times 10^{4}\right.$ cells/chamber $)$ were seeded on the upper of the transwell chambers (Thermo Fisher Scientific, Inc.), DMEM (cat. no. 12430104; Invitrogen; Thermo Fisher Scientific, Inc.) and FBS (10\%) were plated in the lower chamber, and invasion assays were added matrix ( $4^{\circ} \mathrm{C}$, duration of precoating: $30 \mathrm{~min}$ ), Matrigel was used to distinguish between migration and invasion. After $48 \mathrm{~h}$, cells were fixed with $4 \%$ paraformaldehyde $\left(37^{\circ} \mathrm{C}\right.$ and $\left.5 \% \mathrm{CO}_{2}\right)$ for $20 \mathrm{~min}$ and then washed using PBS three times. Subsequently, crystal violet $(0.05 \%$ for $30 \mathrm{~min}$ at room temperature) was used to stain cells. A light microscope was used to observe migratory cells in the lower chamber (Olympus Corporation).

Statistical analysis. All data were analyzed using SPSS 22.0 (IBM, Corp.) and are presented as the mean \pm SD (experiments were repeated in triplicate). One-way ANOVA and Bonferroni's post hoc test was used to compare differences between multiple groups. $\mathrm{P}<0.05$ was considered to indicate a statistically significant difference.

\section{Results}

miR-425 promotes sorafenib resistance in liver cancer. To explore the role of miRNAs in drug resistance in liver cancer,
TCGA database was screened for highly expressed miRNAs, and their enrichment was analyzed in sorafenib-resistant samples from GEO (GSE93595 and GSE94550), there were 48 different overlapping miRNAs (Fig. 1A and Table SI). Among the results, miR-425 was identified to be highly expressed in tumor tissues. In addition, miR-425 has been found to promote liver cancer cell proliferation and migration (4). To explore the association between miR-425 and clinical prognosis, the miRBase database (http://www.mirbase.org/) (26) was used for analysis, revealing that miR-425 was closely associated with poor survival and prognosis in patients with liver cancer (Fig. 1B). Therefore, miR-425 was chosen for further study. miR-425 expression in liver cancer cells was detected by qPCR, and MIHA cells were used as the negative control. The results indicated that miR-425 was abnormally expressed in liver cancer cell lines compared with in MIHA cells $(\mathrm{P}<0.05$; Fig. 1C). miR-425-knockdown cell lines were generated and miR-425 expression was detected after transfection with miR-425 inhibitors (Fig. 1D). To detect the effect of miR-425 on sorafenib sensitivity, miR-425 inhibitors plasmids were transfected in HepG2 cells, revealing that knockdown of miR-425 increased the sensitivity of liver cancer cells to sorafenib (Fig. 1E). Further experiments indicated that miR-425-knockdown decreased the migration and invasion of liver cancer cells (Fig. 1F).

Inhibition of miR-425 promotes lipophagy by regulating autophagy in liver cancer. Since an increase in LDs is associated with chemotherapeutic resistance (27), the present study evaluated whether inhibition of miR-425 could induce fat phagocytosis by decreasing the number of LDs. The HepG2 cell line was chosen to exclude the impact of p53 mutations, since it is p53-wild-type, because p53 has reported to impact LDs (28). The results indicated upregulation of LC3 II (a marker of autophagy induction) after inhibition of miR-425 in HepG2 cells (Fig. 2A). In addition, inhibition of miR-425 significantly decreased the number of LDs (Fig. 2B and C), indicating that miR-425 could regulate lipophagy. Consistent with this observation, immunofluorescence analysis revealed that inhibition of miR-425 significantly induced the colocalization of LDs and LC3 (si-NC was the negative control and miR-425 inhibitors is the inhibitors of miR-425; Fig. 2D and E).

Inhibition of miR-425-induces lipophagy reverses sorafenib resistance. Cancer cells rich in LDs are resistant to chemotherapeutic drugs (27). Therefore, to understand whether lipophagy induced by miR-425 inhibitors is necessary for making liver cancer cells become sensitive to sorafenib-induced cytotoxicity, liver cancer cells were pretreated with BafA1 for $6 \mathrm{~h}$ and cytotoxicity was evaluated. The results revealed that inhibition of autophagy by BafA1 attenuated the synergistic cytotoxicity of miR-425 inhibitors in sorafenib-resistant HepG2 cells (Fig. 3A and B). These results suggested that autophagy induced by miR-425 inhibitors may promote the synergistic effects of standard chemotherapeutic drugs in drug-resistant cells. To further confirm the role of miR-425 in chemoresistance, miR-425 was transiently knocked down in liver cancer cells, and it was confirmed that the synergistic effect between miR-425 inhibitors and sorafenib observed in resistant cells was dependent on autophagy (Fig. 3C and D) (11). 
A
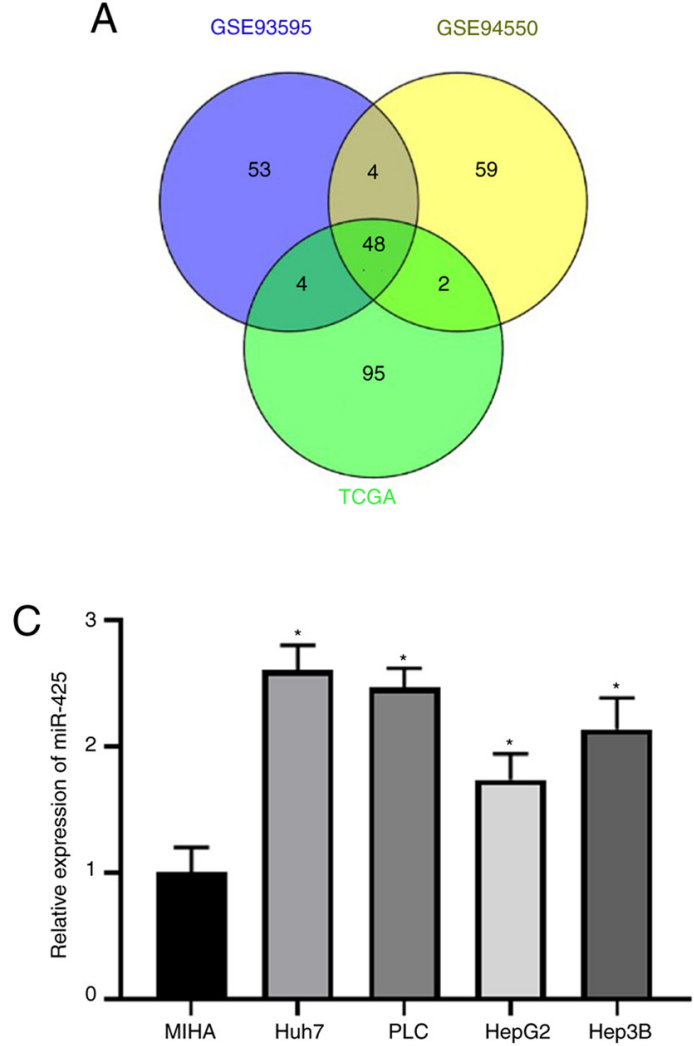

$E$

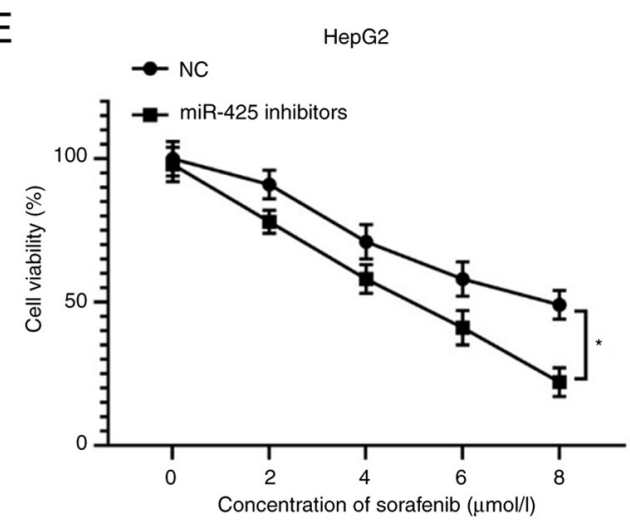

B

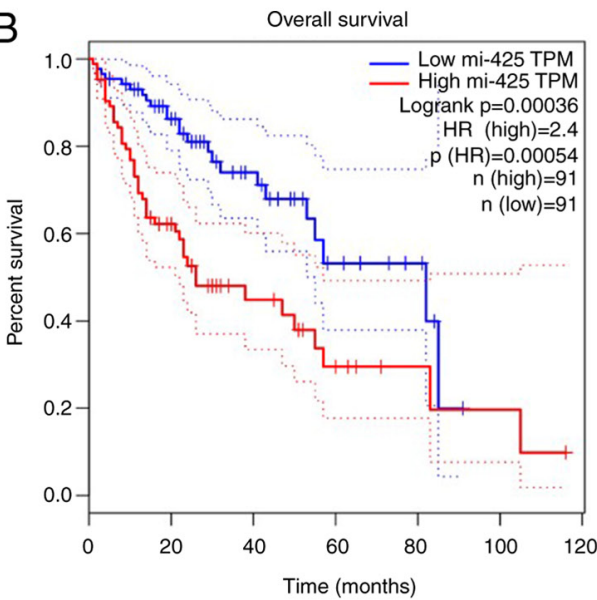

$\mathrm{D}$

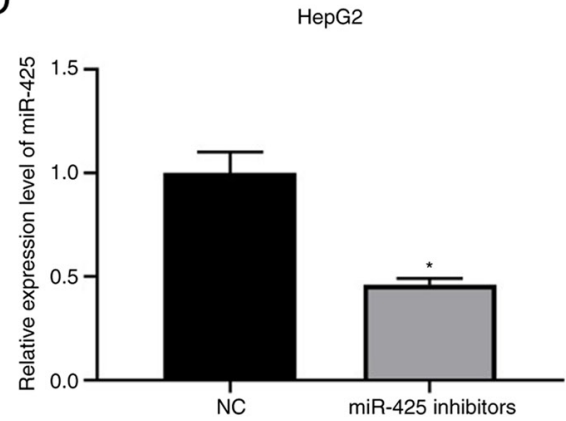

$\mathrm{F}$

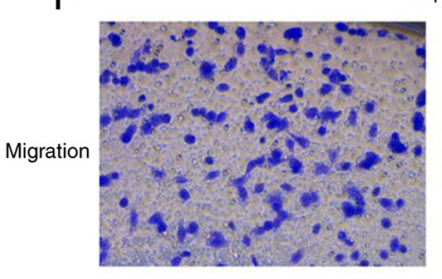

HepG2
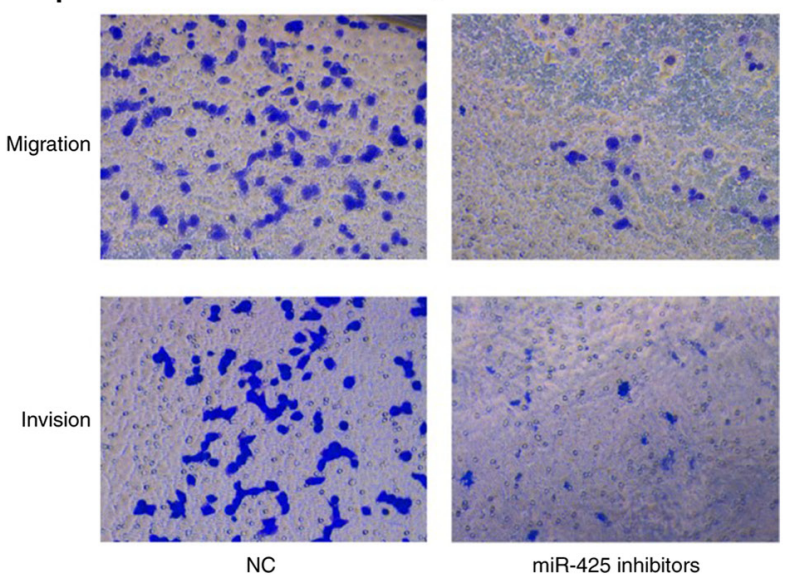

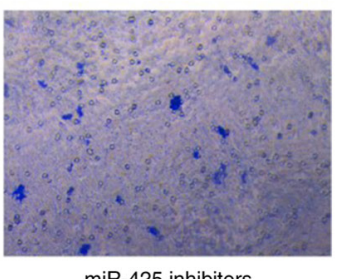

miR-425 inhibitors

Figure 1. (A) Enrichment of highly expressed miRNA genes in sorafenib-resistant samples from the Gene Expression Omnibus database and TCGA datasets. (B) miR-425 expression and clinical prognosis of patients with hepatocellular carcinoma. (C) miR-425 expression in different liver cancer cells was detected by RT-qPCR. (D) miR-425 expression was detected by RT-qPCR after transfection with miR-425 inhibitors. (E) Cell Counting Kit-8 assay was used to evaluate the changes in cell proliferation after miR-425-knockdown. (F) Transwell assays were used to evaluate invasion and migration after miR-425-knockdown (magnification, $\mathrm{x} 400$ ). ${ }^{*} \mathrm{P}<0.05$ vs. MIHA cells or si-NC. RT-qPCR, reverse transcription-quantitative PCR; miR, microRNA; si, small interfering RNA; NC, negative control; TCGA, The Cancer Genome Atlas.

The current results indicated that pretreatment with $25 \mathrm{nM}$ BafA1 for $6 \mathrm{~h}$ resulted in increased resistance to sorafenib in liver cancer cells treated with miR-425 inhibitors compared with the control (Fig. 3E). These results clearly indicate that autophagy induced by miR-425 inhibitors make liver cancer cells sensitive to drug-induced cytotoxicity.

miR-425-knockdown promotes SIRT1 expression. To further explore the targets of miR-425, bioinformatic analysis using StarBase was performed. SIRT1 was identified as a potential direct target of miR-425 (Fig. 4A). The correlation between SIRT1 and miR-425 expression in liver cancer was also analyzed using the StarBase database, revealing a negative correlation between SIRT1 and miR-425 expression (Fig. 4B).

To verify whether miR-425 regulated SIRT1 expression, miR-425 mimics were used to overexpress miR-425 (Fig. 4C). The RT-qPCR results indicated that SIRT1 expression was significantly increased after miR-425-knockdown and significantly decreased after miR-425 overexpression (Fig. 4D), which was consistent with the western blotting 
A
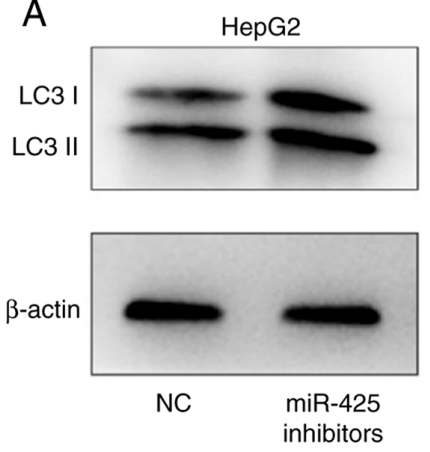

C

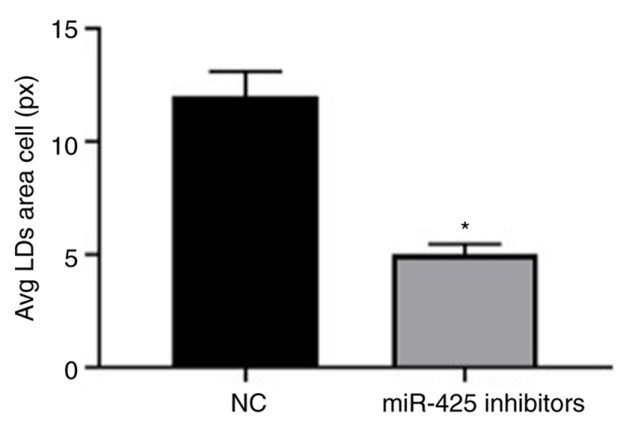

E

NC
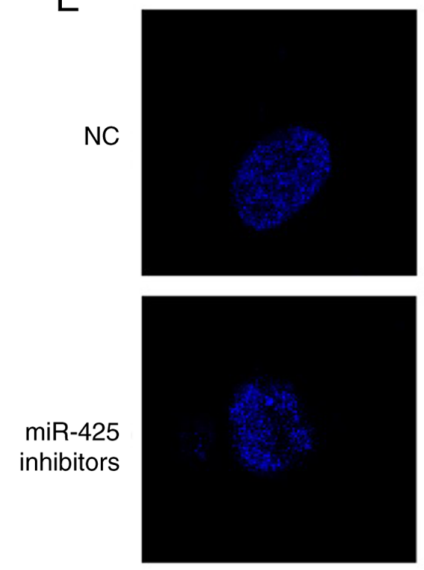

DAPI
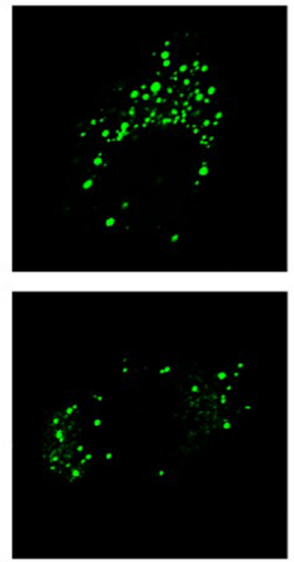

Bodipy
B
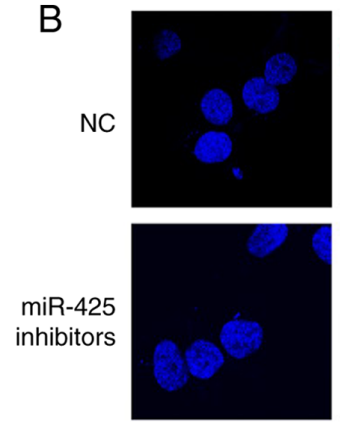

DAPI

D
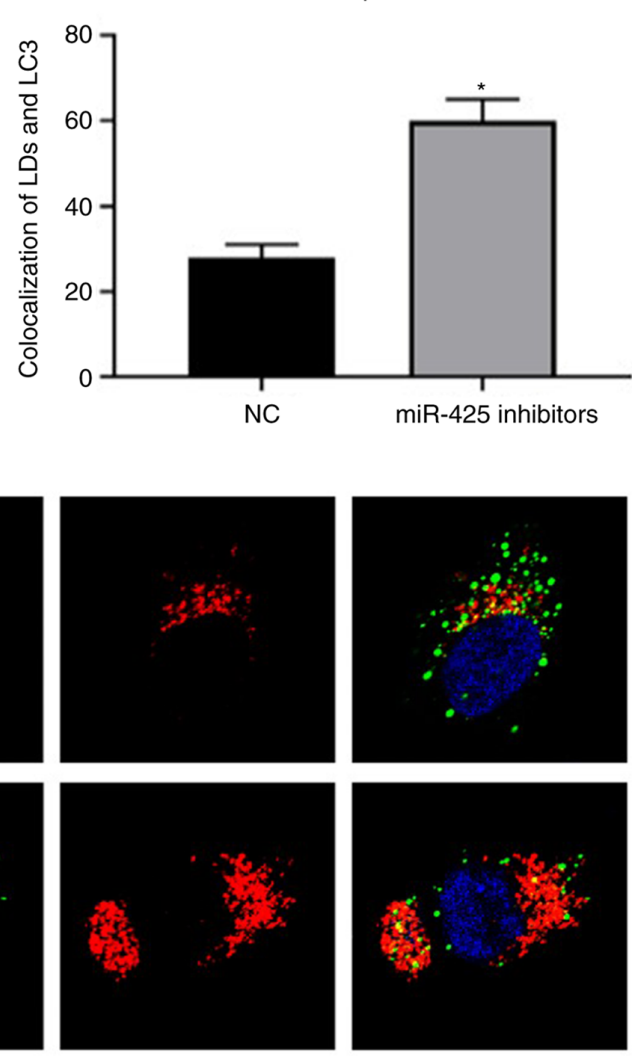

LC3

HepG2

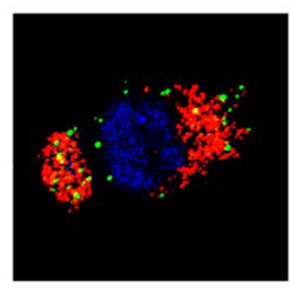

Merge
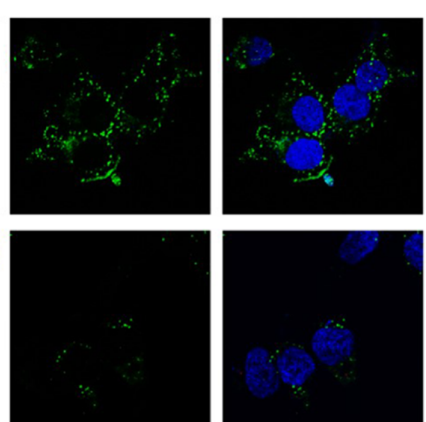

Bodipy

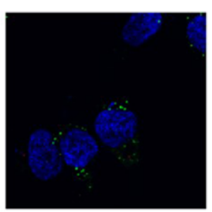

Merge

Figure 2. (A) Western blotting was used to detect LC3 protein expression after miR-425-knockdown. (B and C) Immunofluorescence was used to detect the changes in LDs after miR-425-knockdown. (D and E) Immunofluorescence was used to detect the co-localization of LDs and LC3 after miR-425-knockdown (magnification, $\mathrm{x} 400$ ). ${ }^{*} \mathrm{P}<0.05$ vs. si-NC. miR, microRNA; si, small interfering RNA; NC, negative control; LD, lipid droplet.

results (Fig. 4E). Subsequently, a luciferase reporter assay was used to verify whether miR-425 directly targeted SIRT1. The results revealed that miR-425 significantly inhibited luciferase activity in cells transfected with the wild-type SIRT1-3'UTR plasmid, but had no effect on luciferase activity in cells transfected with the mutant SIRT1-3 UTR plasmid (Fig. 4F). A previous study has reported that adipose triglyceride lipase (ATGL) can regulate SIRT1 to mediate lipophagy (16). Therefore, qPCR was performed in the present study to evaluate ATGL mRNA expression after transfection with miR-425 inhibitors or mimics, revealing that miR-425 modulation did not impact ATGL mRNA expression (Fig. 4G). The current results suggested that miR-425 regulated lipophagy independently of ATGL. Therefore, combining the bioinformatic and experimental results, it was hypothesized that SIRT1 may be a direct target of miR-425.

miR-425 regulates lipophagy via SIRT1. To confirm whether miR-425 regulated lipophagy through SIRT1, si-SIRT1 was used to knock down SIRT1 expression, and SIRT1-knockdown was verified by RT-qPCR and western blot analysis (Fig. 5A and B). Subsequently, the miR-425- and SIRT1-knockdown plasmids were co-transfected into cells, revealing that autophagy and lipophagy were decreased. The level of LDs was significantly increased in co-transfected cells compared with that in cells with only miR-425-knockdown, indicating that LDs were not degraded by autophagy (Fig. 5C and D). Western blot analysis of LC3 indicated that autophagy was inhibited in the co-transfected group compared 
A

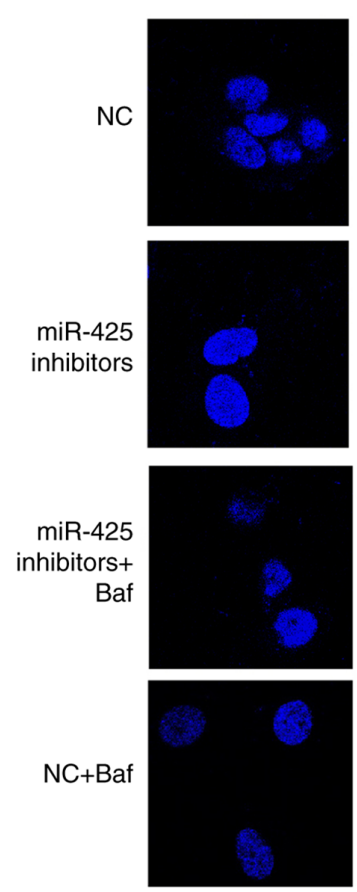

DAPI
HepG2
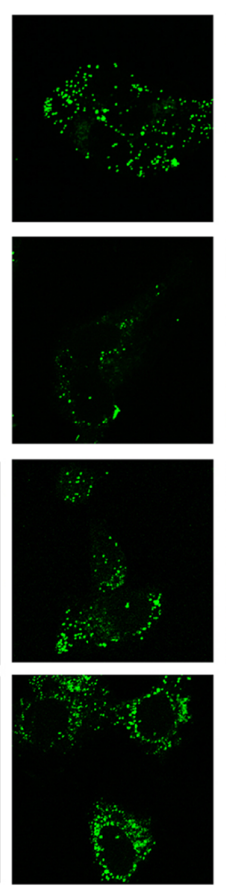

Bodipy
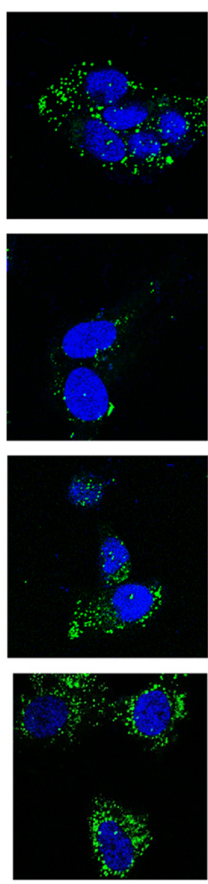

Merge
B

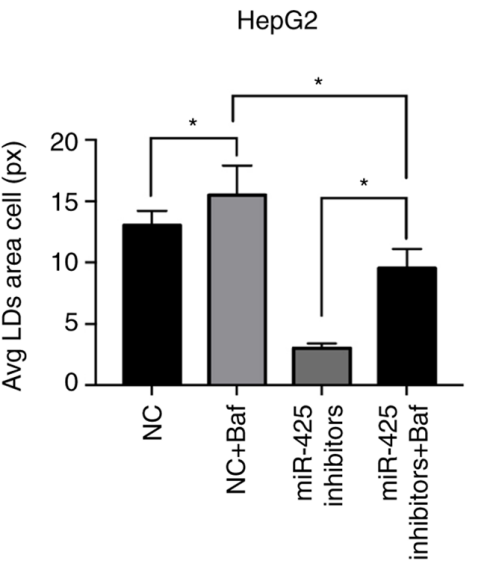

C

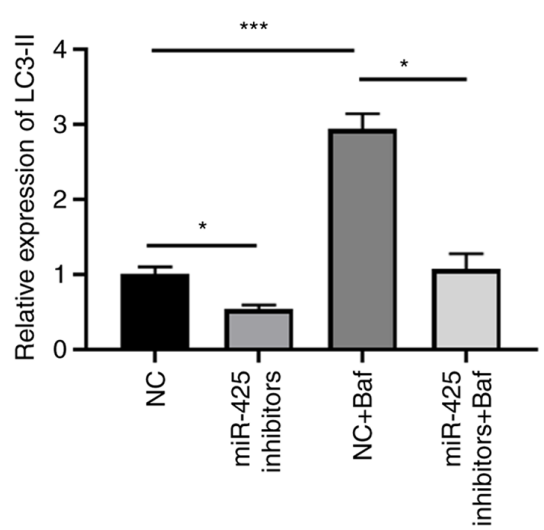

D

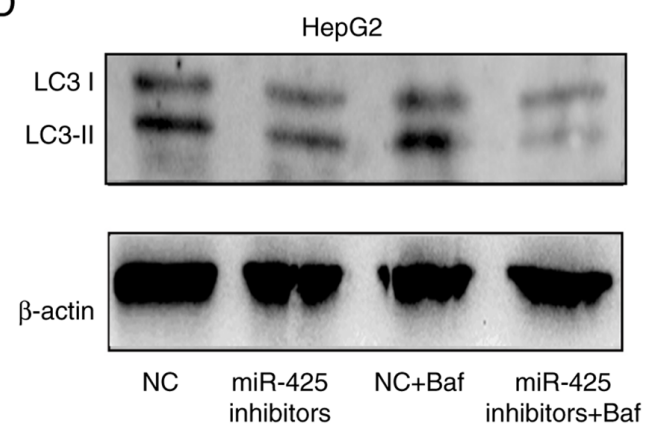

$E$

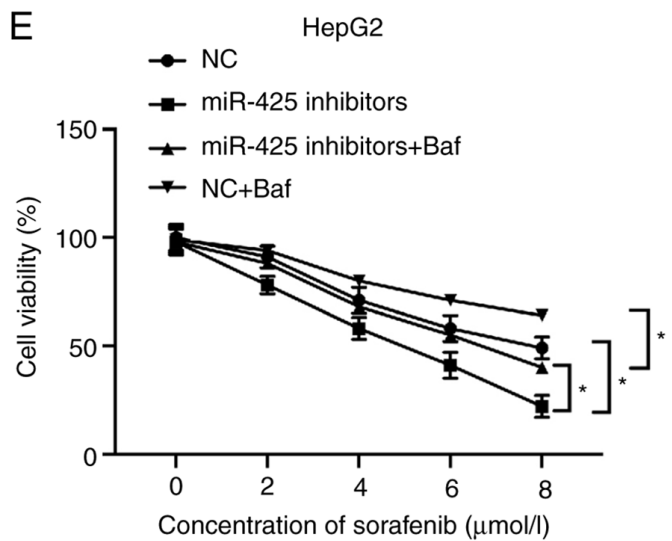

Figure 3. (A and B) Immunofluorescence was used to detect the changes in LDs after inhibition of autophagy. (C and D) Western blotting was used to detect LC3 protein expression after inhibition of autophagy. (E) Cell Counting Kit-8 assay was used to evaluate the changes in cell proliferation after inhibition of autophagy (magnification, $\mathrm{x} 400$ ). ${ }^{*} \mathrm{P}<0.05 ;{ }^{* * *} \mathrm{P}<0.001$. miR, microRNA; si, small interfering RNA; NC, negative control; LD, lipid droplet; Baf, Bafilomycin A.

with the miR-425-knockdown group, which confirmed the inhibition of lipophagy (Fig. 5E and F).

\section{Discussion}

Currently, the treatment of liver cancer mainly includes surgical resection, radiotherapy and chemotherapy, but the effects are unsatisfactory $(1,2)$. Although sorafenib is an effective drug for the treatment of advanced liver cancer, the benefit that patients receive from sorafenib treatment is limited due to the development of drug resistance (3). Therefore, there is an urgent need for further research on drug resistance. LDs are spherical organelles with phospholipid monolayer membrane structures that were initially identified as the main components dynamically responding to the needs of cell energy metabolism and regulating the storage of lipid molecules, triglycerides, cholesterol esters and small amounts of vitamins (27). In recent years, with the in-depth understanding of cellular lipid metabolism and LD function, the role of LDs in drug resistance has been increasingly recognized (11). Studies have demonstrated that stearoyl-CoA desaturase is highly expressed in various types of tumors and is associated with cell membrane fluidity and chemotherapeutic resistance (29-32). In fact, an increase in LDs is associated with drug resistance in tumor cells, and this association has been confirmed in breast, prostate and ovarian cancer (11). In addition, the expression of components of cancer stem cell marker signaling pathways 
A

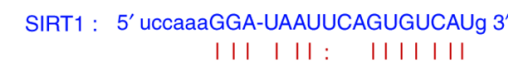

miR-425: $3^{\prime}$ aguugcCCUCACUAG--CACAGUAa 5
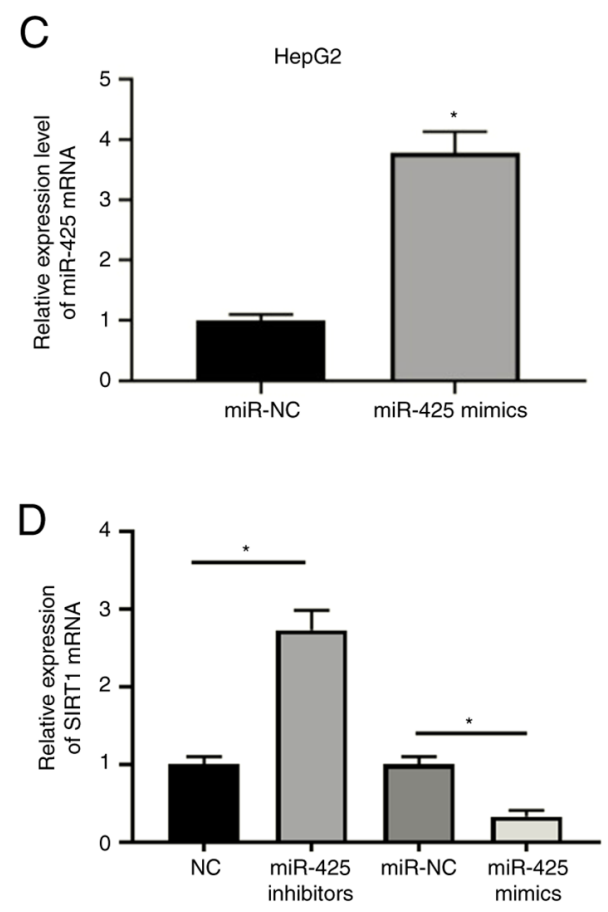

$\mathrm{F}$

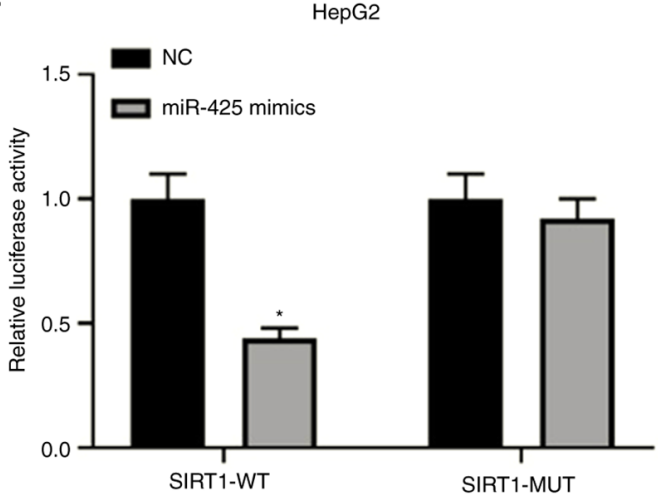

$\mathrm{B}$

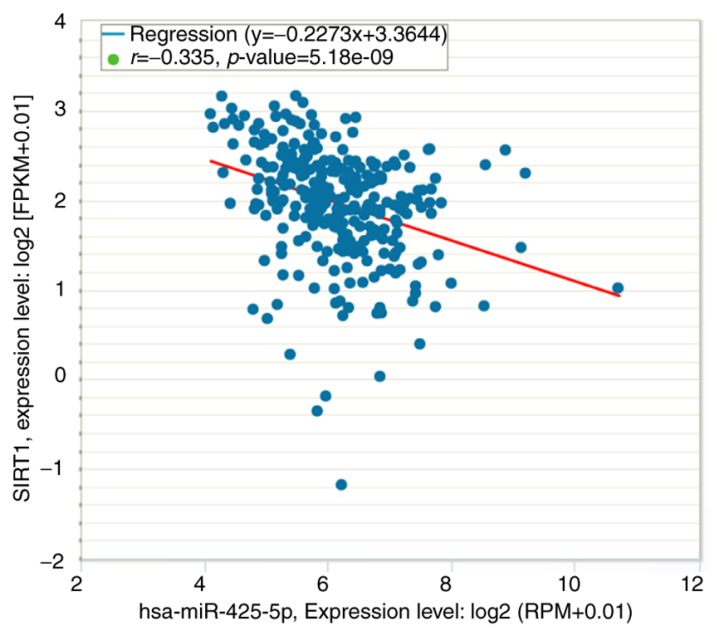

$E$
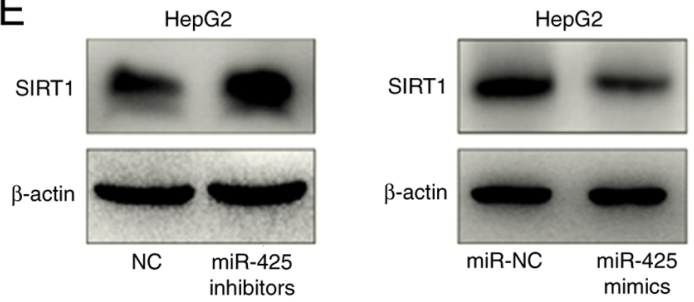

G

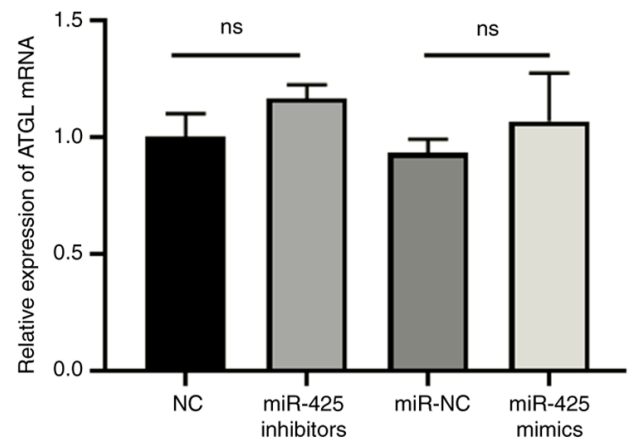

Figure 4. (A) Bioinformatic analysis revealed the binding sites of SIRT1 and miR-425. (B) Bioinformatic analysis revealed the correlation between SIRT1 and miR-425 expression. (C) miR-425 expression was detected by RT-qPCR after transfection with miR-425 mimics. SIRT1 expression was detected by (D) RT-qPCR and (E) western blotting after miR-425-knockdown or overexpression. (F) Relative luciferase activity was used to detect the binding between miR-425 and SIRT1. (G) ATGL was detected after modulation of miR-425 expression. " $\mathrm{P}<0.05$ vs. NC. miR, microRNA; si, small interfering RNA; NC, negative control; SIRT1, silent information regulator 2 homolog 1; WT, wild-type; MUT, mutant; RT-qPCR, reverse transcription-quantitative PCR.

is associated with LDs in several types of cancer, such as glioma, colorectal cancer and HCC (33).

Autophagy serves an important role in malignant biological behaviors, such as tumor proliferation, invasion, metastasis and drug resistance (4). An increasing number of studies have shown that autophagy is closely associated with the development of tumors $(5,34)$. Interfering with the autophagy level in tumor cells is a new idea for tumor therapy (5). For example, Atg7 inhibitors and docetaxel can be used in combination to treat breast cancer (34). In liver cancer, SIRT1 is the only overexpressed member of the SIRT family and is considered to be essential for all stages of liver cancer tumorigenesis (35). In addition, SIRT1 can modulate lipophagy (16), which is the process of autophagic degradation of LDs (35). By decomposing intracellular lipids from LDs into fatty acids, lipid phagocytosis provides energy for cells as well as regulates other cellular processes, such as activation of carcinogenic signaling pathways and drug resistance (11). Considering the important role of LDs and autophagy in liver cancer, lipophagy may be a factor promoting the process of drug resistance in liver cancer.

In the present study, mRNA sequencing data for patients with hepatocellular carcinoma in TCGA database and for sorafenib-resistant samples in the GEO database were compared to identify miR-425, and it was revealed that miR-425 was associated with a poor prognosis. Therefore, a cell line with low miR-425 expression was generated through 
A
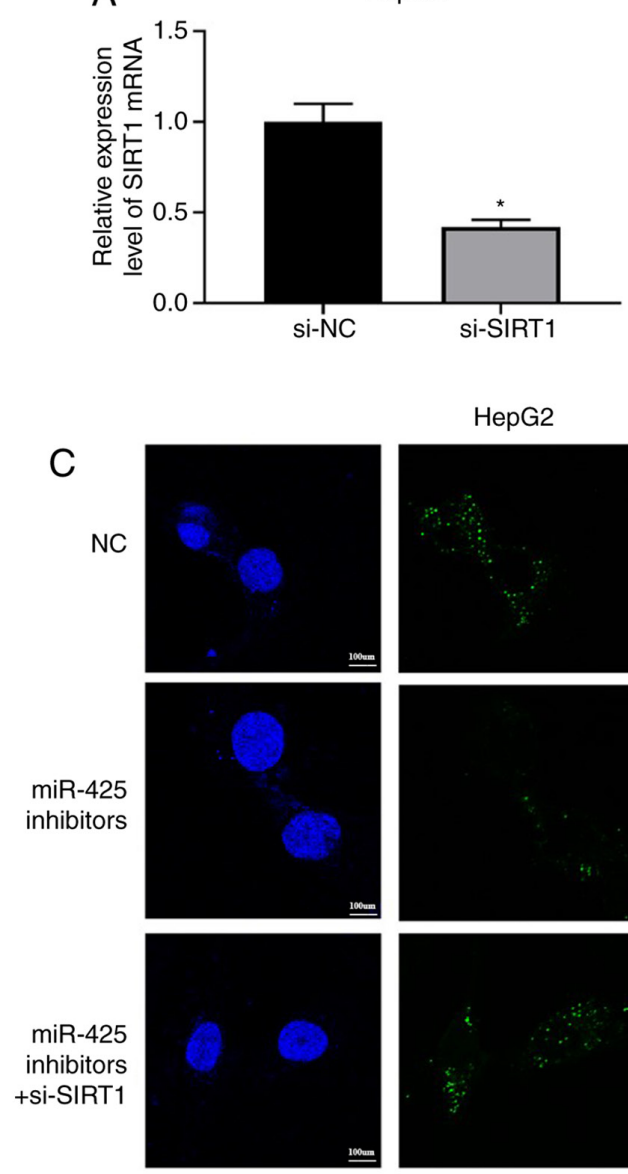

E
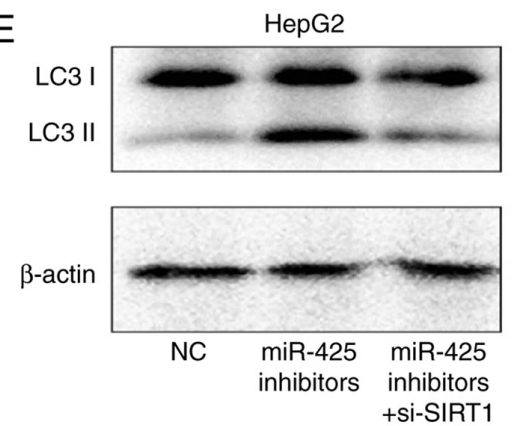

B

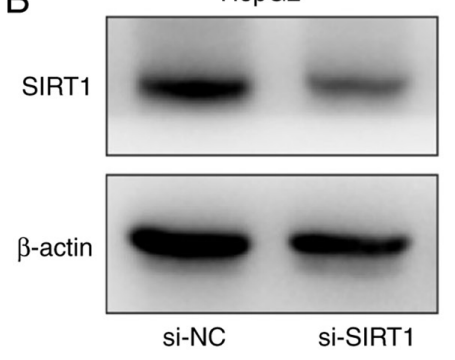

$\mathrm{D}$

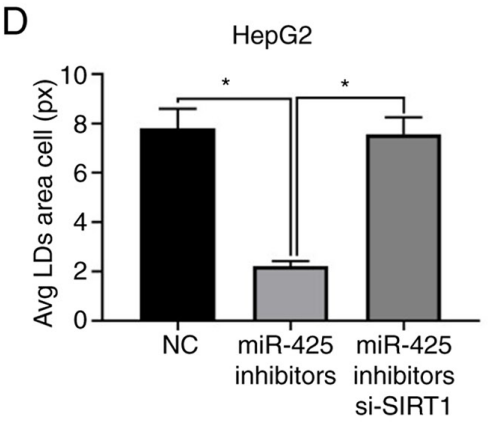

$\mathrm{F}$

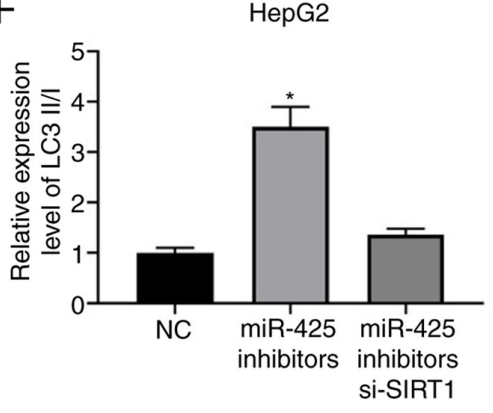

Figure 5. SIRT1 expression was detected by (A) reverse transcription-quantitative PCR and (B) western blotting after transfection with si-SIRT1. (C and D) Immunofluorescence was used to detect the changes in LDs after co-transfection with miR-425 inhibitors and si-SIRT1. (E and F) Western blotting was used to detect LC3 protein expression after co-transfection with miR-425 inhibitors and si-SIRT1. Scale bar, $100 \mu \mathrm{m}$. ${ }^{*} \mathrm{P}<0.05$ vs. si-NC. miR, microRNA; si, small interfering RNA; NC, negative control; LD, lipid droplet; SIRT1, silent information regulator 2 homolog 1.

transfection and the biological function of miR-425 was analyzed. It has been previously found that miR- 425 regulated the proliferation, invasion and metastasis of hepatocellular carcinoma cells (36). These results are consistent with the previous findings that miR- 425 can improve cell survival and is associated with a poor outcome $(37,38)$. Overexpression of miR-425 promotes cell proliferation and migration via targeting RNF11 in HCC and it can also bind with FOXD3 to promote cell migration and invasion $(36,37)$. These results suggested that miR-425 is an oncogene to promote tumor progression. However, relatively few studies have investigated lipophagy and sofafenib resistance in HCC. In the present study, western blot analysis and immunofluorescence experiments were used to evaluate the level of lipophagy. The results revealed that the level of lipophagy decreased after miR-425 inhibition. Additionally, it was found that miR-425 regulated the drug resistance of liver cancer cells through lipophagy. By further studying the mechanism by which miR-425 regulated drug resistance, it was revealed that SIRT1 expression, a key protein in the process of lipophagy, was inhibited after miR-425 inhibition, and rescue experiments demonstrated that miR-425 regulated lipophagy through SIRT1. A previous study demonstrated that SIRT1 expression was high in HCC cell lines, including HepG2 and Hep3B cells, and its upregulation may result in increased cell proliferation (39). The current study identified a specific mechanism of miR-425 on SIRT1-associated lipophagy, miR-425 inhibitors can increase SIRT1 expression to induce lipophagy which is consistent with 
previous findings (16). The aforementioned results indicated the important role and related mechanism of miR-425 in lipophagy and drug resistance in liver cancer, and suggested that miR-425 may become a new target for tumor therapy.

\section{Acknowledgements}

Not applicable.

\section{Funding}

The present study was supported by the National Natural Science Foundation of China (grant nos. 81871983 and 82003040), the Natural Science Foundation of Liaoning Province (grant no. LQNK201719) and the Natural Science Foundation of Liaoning Province (grant no. 2020-BS-103).

\section{Availability of data and materials}

The datasets generated and/or analyzed during the current study are available in the Gene Expression Omnibus (https://ncbi.nlm.nih.gov/geo) repository.

\section{Authors' contributions}

GS and LY designed the study and performed the experiments. HJ, SW and BL analyzed the biological data. LY revised the manuscript. HL conceived the study and participated in manuscript writing and revisions. GS and LY were responsible for confirming the authenticity of the raw data. All authors have read and approved the final manuscript.

\section{Ethics approval and consent to participate}

Not applicable.

\section{Patient consent for publication}

Not applicable.

\section{Competing interests}

The authors declare that they have no competing interests.

\section{References}

1. Chen Z, Xie H, Hu M, Huang T, Hu Y, Sang N and Zhao Y: Recent progress in treatment of hepatocellular carcinoma. Am J Cancer Res 10: 2993-3036, 2020.

2. Zhu ZX, Huang JW, Liao MH and Zeng Y: Treatment strategy for hepatocellular carcinoma in China: Radiofrequency ablation versus liver resection. Jpn J Clin Oncol 46: 1075-1080, 2016.

3. Perera S, Kelly D and O'Kane GM: Non-immunotherapy options for the first-line management of hepatocellular carcinoma: Exploring the evolving role of sorafenib and lenvatinib in advanced disease. Curr Oncol 27 (Suppl 3): S165-S172, 2020.

4. White E, Mehnert JM and Chan CS: Autophagy, metabolism, and cancer. Clin Cancer Res 21: 5037-5046, 2015.

5. Li YJ, Lei YH, Yao N, Wang CR, Hu N, Ye WC, Zhang DM and Chen ZS: Autophagy and multidrug resistance in cancer. Chin J Cancer 36: 52, 2017.

6. Zhang Z, Yao Z, Chen Y, Qian L, Jiang S, Zhou J, Shao J, Chen A, Zhang F and Zheng S: Lipophagy and liver disease: New perspectives to better understanding and therapy. Biomed Pharmacother 97: 339-348, 2018.
7. Maan M,Peters JM, Dutta M and Patterson AD: Lipid metabolism and lipophagy in cancer. Biochem Biophys Res Commun 504: 582-589, 2018.

8. Madrigal-Matute J and Cuervo AM: Regulation of liver metabolism by autophagy. Gastroenterology 150: 328-339, 2016.

9. Beloribi-Djefaflia S, Vasseur S and Guillaumond F: Lipid metabolic reprogramming in cancer cells. Oncogenesis 5: e189, 2016.

10. Dong $\mathrm{H}$ and Czaja MJ: Regulation of lipid droplets by autophagy. Trends Endocrinol Metab 22: 234-240, 2011.

11. Mondal S, Roy D, Sarkar Bhattacharya S, Jin L, Jung D, Zhang S, Kalogera E, Staub J, Wang Y, Xuyang W, et al: Therapeutic targeting of PFKFB3 with a novel glycolytic inhibitor PFK158 promotes lipophagy and chemosensitivity in gynecologic cancers. Int J Cancer 144: 178-189, 2019.

12. Li X, Zhou Y, Yang L, Ma Y, Peng X, Yang S, Li H and Liu J: LncRNA NEAT1 promotes autophagy via regulating miR-204/ ATG3 and enhanced cell resistance to sorafenib in hepatocellular carcinoma. J Cell Physiol 235: 3402-3413, 2020.

13. Yang S, Wang M, Yang L, Li Y, Ma Y, Peng X, Li X, Li B, Jin H and Li H: MicroRNA-375 Targets ATG14 to inhibit autophagy and sensitize hepatocellular carcinoma cells to sorafenib. Onco Targets Ther 13: 3557-3570, 2020.

14. Lapierre LR, Kumsta C, Sandri M, Ballabio A and Hansen M: Transcriptional and epigenetic regulation of autophagy in aging. Autophagy 11: 867-880, 2015.

15. Maiese K: Targeting the core of neurodegeneration: FoxO, mTOR, and SIRT1. Neural Regen Res 16: 448-455, 2021.

16. Sathyanarayan A, Mashek MT and Mashek DG: ATGL promotes autophagy/lipophagy via SIRT1 to control hepatic lipid droplet catabolism. Cell Rep 19: 1-9, 2017.

17. Masters JR, Thomson JA, Daly-Burns B, Reid YA, Dirks WG, Packer P, Toji LH, Ohno T, Tanabe H, Arlett CF, et al: Short tandem repeat profiling provides an international reference standard for human cell lines. Proc Natl Acad Sci USA 98: 8012-8017, 2001

18. Li HR, Shagisultanova EI, Yamashita K, Piao Z, Perucho M and Malkhosyan SR: Hypersensitivity of tumor cell lines with microsatellite instability to DNA double strand break producing chemotherapeutic agent bleomycin. Cancer Res 64: 4760-4767, 2004.

19. Livak KJ and Schmittgen TD: Analysis of relative gene expression data using realtime quantitative PCR and the 2(-Delta Delta C(T)) method. Methods 25: 402-408, 2001.

20. Ohata Y, Shimada S, Akiyama Y, Mogushi K, Nakao K, Matsumura S, Aihara A, Mitsunori Y, Ban D, Ochiai T, et al: Acquired resistance with epigenetic alterations under Long-Term antiangiogenic therapy for hepatocellular carcinoma. Mol Cancer Ther 16: 1155-1165, 2017.

21. Regan-Fendt K, Li D, Reyes R, Yu L, Wani NA, Hu P, Jacob ST, Ghoshal K, Payne PRO and Motiwala T: Transcriptomics-Based drug repurposing approach identifies novel drugs against Sorafenib-Resistant hepatocellular carcinoma. Cancers (Basel) 12: 2730, 2020.

22. Pu Z,Zhu Y, Wang X, Zhong Y,Peng F and Zhang Y: Identification of prognostic biomarkers and correlation with immune infiltrates in hepatocellular carcinoma based on a competing endogenous RNA network. Front Genet 12: 591623, 2021.

23. Fang Q, Li T, Chen $\mathrm{P}$, Wu Y, Wang T, Mo L, Ou J and Nandakumar KS: Comparative analysis on abnormal methylome of differentially expressed genes and disease pathways in the immune cells of RA and SLE. Front Immunol 12: 668007, 2021.

24. Shi Z, Kong X, Li C, Liu H, Aliagan AI, Liu L, Shi Y, Shi X, Ma B, Jin R, et al: Bioinformatic analysis of differentially expressed genes as prognostic markers in pheochromocytoma and paraganglioma tumors. Genes Genet Syst 96: 55-69, 2021.

25. Lu M, Fan X, Liao W, Li Y, Ma L, Yuan M, Gu R, Wei Z, Wang C and Zhang $\mathrm{H}$ : Identification of significant genes as prognostic markers and potential tumor suppressors in lung adenocarcinoma via bioinformatical analysis. BMC Cancer 21: 616, 2021.

26. Kozomara A, Birgaoanu M and Griffiths-Jones S: MiRBase: From microRNA sequences to function. Nucleic Acids Res 47: D155-D162, 2019.

27. Martinez-Lopez N and Singh R: Autophagy and lipid droplets in the liver. Annu Rev Nutr 35: 215-237, 2015.

28. Thai SF, Jones CP, Robinette BL, Ren H, Vallanat B, Fisher AA and Kitchin KT: Effects of silver nanoparticles and silver nitrate on mRNA and microRNA expression in human hepatocellular carcinoma cells (HepG2). J Nanosci Nanotechnol 21: 5414-5428, 2021. 
29. Noto A, De Vitis C, Pisanu ME, Roscilli G, Ricci G, Catizone A, Sorrentino G, Chianese G, Taglialatela-Scafati O, Trisciuoglio D, et al: Stearoyl-CoA-desaturase 1 regulates lung cancer stemness via stabilization and nuclear localization of YAP/TAZ. Oncogene 36: 4573-4584, 2017.

30. Kikuchi $\mathrm{K}$ and Tsukamoto $\mathrm{H}$ : Stearoyl-CoA desaturase and tumorigenesis. Chem Biol Interact 316: 108917, 2020.

31. Tesfay L, Paul BT, Konstorum A, Deng Z, Cox AO, Lee J, Furdui CM, Hegde P, Torti FM and Torti SV: Stearoyl-CoA Desaturase 1 protects ovarian cancer cells from ferroptotic cell death. Cancer Res 79: 5355-5366, 2019.

32. Pisanu ME, Noto A, De Vitis C, Morrone S, Scognamiglio G, Botti G, Venuta F, Diso D, Jakopin Z, Padula F, et al: Blockade of Stearoyl-CoA-desaturase 1 activity reverts resistance to cisplatin in lung cancer stem cells. Cancer Lett 406: 93-104, 2017.

33. Yi M, Li J, Chen S, Cai J, Ban Y, Peng Q, Zhou Y, Zeng Z, Peng S, Li X, et al: Emerging role of lipid metabolism alterations in cancer stem cells. J Exp Clin Cancer Res 37: 118, 2018.

34. Gong C, Hu C, Gu F, Xia Q, Yao C, Zhang L, Qiang L, Gao S and Gao Y: Co-delivery of autophagy inhibitor ATG7 siRNA and docetaxel for breast cancer treatment. J Control Release 266: 272-286, 2017.

35. Xiong H, Ni Z, He J, Jiang S, Li X, He J, Gong W, Zheng L, Chen S, Li B, et al: LncRNA HULC triggers autophagy via stabilizing Sirt1 and attenuates the chemosensitivity of HCC cells. Oncogene 36: 3528-3540, 2017.
36. Wu H, Shang J, Zhan W, Liu J, Ning H and Chen N: MiR4255p promotes cell proliferation, migration and invasion by directly targeting FOXD3 in hepatocellular carcinoma cells. Mol Med Rep 20: 1883-1892, 2019.

37. Rao D, Guan S, Huang J, Chang Q and Duan S: MiR-425-5p acts as a molecular marker and promoted proliferation, migration by targeting RNF11 in hepatocellular carcinoma. Biomed Res Int 2020: 6530973, 2020

38. Vaira V, Roncalli M, Carnaghi C, Faversani A, Maggioni M, Augello C, Rimassa L, Pressiani T, Spagnuolo G, Di Tommaso L, et al: MicroRNA-425-3p predicts response to sorafenib therapy in patients with hepatocellular carcinoma. Liver Int 35: 1077-1086, 2015

39. Portmann S, Fahrner R, Lechleiter A, Keogh A, Overney S, Laemmle A, Mikami K, Montani M, Tschan MP, Candinas D and Stroka D: Antitumor effect of SIRT1 inhibition in human HCC tumor models in vitro and in vivo. Mol Cancer Ther 12: 499-508, 2013

This work is licensed under a Creative Commons Attribution-NonCommercial-NoDerivatives 4.0 International (CC BY-NC-ND 4.0) License. 